\title{
General two-mode squeezed states
}

\author{
R.F. Bishop ${ }^{1}$ and A. Vourdas ${ }^{2 *}$ \\ ${ }^{1}$ Department of Mathematics, University of Manchester, Institute of Science and \\ Technology, Manchester, United Kingdom \\ ${ }^{2}$ Fachbereich Physik, Universität Marburg, Federal Republic of Germany
}

Received October 16, 1987

\begin{abstract}
The states $\hat{\theta}\left|A_{1} A_{2}\right\rangle$ are considered, where the operators $\hat{\theta}$ are associated with a unitary representation of the group $S p(4, \mathbb{R})$, and the two-mode Glauber coherent states $\left|A_{1} A_{2}\right\rangle$ are joint eigenstates of the destruction operators $a_{1}$ and $a_{2}$ for the two independent oscillator modes. We show that they are ordinary coherent states with respect to new operators $b_{1}$ and $b_{2}$, which are themselves general linear (Bogoliubov) transformations of the original operators $a_{1}, a_{2}$ and their hermitian conjugates $a_{1}^{\dagger}, a_{2}^{\dagger}$. We further show how they may be regarded as the most general two-mode squeezed states. Most previous work on two-mode squeezed states appears to be based on more restrictive definitions than our own, and thereby reduces to special cases which are unified within our treatment.
\end{abstract}

We first consider the annihilation and creation operators $a$ and $a^{\dagger}$ associated with a single oscillator mode, and which obey the usual canonical commutation relation,

$\left[a, a^{\dagger}\right]=1$.

The one-mode squeezed states associated with these operators are by now well-known (see e.g. [1-6]). In terms of the vacuum state $|0\rangle$, defined as usual by $a|0\rangle=0$, they are defined as,

$|A ; \rho \theta \lambda\rangle \equiv U_{2}(\rho \theta \lambda)|A\rangle \equiv U_{2}(\rho \theta \lambda) U_{1}(A)|0\rangle$

where the displacement operator, $U_{1}(A)$, given by

$U_{1}(A) \equiv \exp \left(A a^{\dagger}-A^{*} a\right), \quad A \in \mathbb{C}$

and the squeezing operator, $U_{2}(\rho \theta \lambda)$, given by

$U_{2}(\rho \theta \lambda) \equiv \exp \left(-\frac{1}{4} \rho \mathrm{e}^{-\mathrm{i} \theta} a^{\dagger 2}+\frac{1}{4} \rho \mathrm{e}^{\mathrm{i} \theta} a^{2}\right) \exp \left(\mathrm{i} \lambda a^{\dagger} a\right)$

$\rho, \theta, \lambda \in \mathbb{R}, \quad \rho>0, \quad 0<\theta<2 \pi$,

* Present address: Department of Electrical Engineering and Electronics, University of Liverpool, Brownlow Hill, P.O. Box 147, Liverpool L69 3BX, United Kingdom are unitary representations of respectively the Weyl group and the group $S U(1,1)$. These squeezed coherent states are just ordinary coherent states with respect to the operators $b$ and $b^{\dagger}$,

$$
\begin{aligned}
U_{2} a U_{2}^{\dagger} & \equiv b=\gamma a+\delta a^{\dagger}, \\
U_{2} a^{\dagger} U_{2}^{\dagger} & \equiv b^{\dagger}=\delta^{*} a+\gamma^{*} a^{\dagger} \\
\gamma & \equiv \mathrm{e}^{-\mathrm{i} \lambda} \cosh \left(\frac{1}{2} \rho\right), \quad \delta \equiv \mathrm{e}^{-\mathrm{i}(\lambda+\theta)} \sinh \left(\frac{1}{2} \rho\right)
\end{aligned}
$$

which are linear (Bogoliubov) transformations of the original operators $a$ and $a^{\dagger}$. The transformation is easily seen from (1) and (5) to be a canonical one,

$\left[b, b^{\dagger}\right]=1$.

Two-mode squeezed states were first considered as trivial products of two one-mode squeezed states,

$$
\begin{aligned}
& \left|A_{1} A_{2} ; \rho_{1} \theta_{1} \lambda_{1}, \rho_{2} \theta_{2} \lambda_{2}\right\rangle \equiv \\
& \quad U_{2}^{(1)}\left(\rho_{1} \theta_{1} \lambda_{1}\right) U_{2}^{(2)}\left(\rho_{2} \theta_{2} \lambda_{2}\right)\left|A_{1} A_{2}\right\rangle,
\end{aligned}
$$

where $\left|A_{1} A_{2}\right\rangle \equiv\left|A_{1}\right\rangle\left|A_{2}\right\rangle$ are two-mode Glauber coherent states associated with two independent modes described respectively in terms of operators $a_{1}$ and $a_{2}$ (and their hermitian conjugates), and where $U_{2}^{(i)}\left(\rho_{i} \theta_{i} \lambda_{i}\right), i=1,2$ are the squeezing operators given in (4) in terms of annihilation and creation operators $a_{i}$ 
and $a_{i}^{\dagger}$. In fact, Milburn [7] proved that a certain particular definition of two-mode squeezing, leads precisely to the states defined in (7) as the most trivial generalisation of the one-mode squeezed states.

Caves and Schumaker [8] later adopted a different definition of two-mode squeezing and studied the associated squeezed states. More recently we have ourselves shown [9] that these latter squeezed states are based on the so-called discrete series representation of the group $S U(1,1)$. Both this type of squeezing and also a separate type of squeezing based on the so-called Schwinger representation of the $S U(2)$ group, have been used recently both in the study of interferometers [10] and in other applications in quantum optics [11]. It is one of our purposes in the present paper to show that all of these different types of twomode squeezing which have been considered separately in the literature, may be unified within our treatment which is based on the larger group $S p(4, \mathbb{R})$.

In terms of the annihilation and creation operators for the two independent modes, which obey the usual canonical commutation relations,

$\left[a_{1}, a_{1}^{\dagger}\right]=1=\left[a_{2}, a_{2}^{\dagger}\right]$

$\left[a_{1}, a_{2}\right]=\left[a_{1}, a_{2}^{\dagger}\right]=\left[a_{1}^{\dagger}, a_{2}\right]=\left[a_{1}^{\dagger}, a_{2}^{\dagger}\right]=0$,

we consider each of the ten possible pairing operators,

$K_{+}^{(1)} \equiv \frac{1}{2} a_{1}^{\dagger 2}, \quad K_{-}^{(1)} \equiv \frac{1}{2} a_{1}^{2}, \quad K_{0}^{(1)} \equiv \frac{1}{2} a_{1}^{\dagger} a_{1}+\frac{1}{4}$

$K_{+}^{(2)} \equiv \frac{1}{2} a_{2}^{\ddagger 2}, \quad K_{-}^{(2)} \equiv \frac{1}{2} a_{2}^{2}, \quad K_{0}^{(2)} \equiv \frac{1}{2} a_{2}^{\dagger} a_{2}+\frac{1}{4}$

$L_{+} \equiv a_{1}^{\dagger} a_{2}^{\dagger}, L_{-} \equiv a_{1} a_{2}, J_{+} \equiv a_{1}^{\dagger} a_{2}, J_{-} \equiv a_{1} a_{2}^{\dagger}$,

which satisfy the $S p(4, \mathbb{R})$ algebra. From (8) and (9) it is a simple business to verify the commutation relations,

$$
\begin{aligned}
& {\left[K_{0}^{(1)}, K_{ \pm}^{(1)}\right]= \pm K_{ \pm}^{(1)}, \quad\left[K_{-}^{(1)}, K_{+}^{(1)}\right]=2 K_{0}^{(1)}} \\
& {\left[K_{0}^{(2)}, K_{ \pm}^{(2)}\right]= \pm K_{ \pm}^{(2)}, \quad\left[K_{-}^{(2)}, K_{+}^{(2)}\right]=2 K_{0}^{(2)}} \\
& {\left[L_{-}, L_{+}\right]=\quad\left[J_{+}, J_{-}\right]=} \\
& 2\left(K_{0}^{(1)}+K_{0}^{(2)}\right), \quad 2\left(K_{0}^{(1)}-K_{0}^{(2)}\right) \\
& {\left[K_{+}^{(1)}, L_{-}\right]=-\frac{1}{2} J_{+}, \quad\left[K_{+}^{(1)}, J_{-}\right]=-\frac{1}{2} L_{+}} \\
& {\left[K_{-}^{(1)}, L_{+}\right]=\frac{1}{2} J_{-}, \cdot \quad\left[K_{-}^{(1)}, J_{+}\right]=\frac{1}{2} L_{-}} \\
& {\left[K_{+}^{(2)}, L_{-}\right]=-\frac{1}{2} J_{-}, \quad\left[K_{+}^{(2)}, J_{+}\right]=-\frac{1}{2} L_{+}} \\
& {\left[K_{-}^{(2)}, L_{+}\right]=\frac{1}{2} J_{+}, \quad\left[K_{-}^{(2)}, J_{-}\right]=\frac{1}{2} L_{-}} \\
& {\left[K_{0}^{(1)}, L_{ \pm}\right]= \pm \frac{1}{2} L_{ \pm}, \quad\left[K_{0}^{(1)}, J_{ \pm}\right]= \pm \frac{1}{2} J_{ \pm}} \\
& {\left[K_{0}^{(2)}, L_{ \pm}\right]= \pm \frac{1}{2} L_{ \pm}, \quad\left[K_{0}^{(2)}, J_{ \pm}\right]=\mp \frac{1}{2} J_{ \pm}} \\
& {\left[L_{+}, J_{+}\right]=-2 K_{+}^{(1)}, \quad\left[L_{-}, J_{+}\right]=2 K_{-}^{(2)}} \\
& {\left[L_{+}, J_{-}\right]=-2 K_{+}^{(2)}, \quad\left[L_{-}, J_{-}\right]=2 K_{-}^{(1)},}
\end{aligned}
$$

and that all other commutators are zero.

We see immediately that the three operators $K_{+}^{(1)}$, $K_{-}^{(1)}$ and $K_{0}^{(1)}$ close under commutation into a subalgebra of $S p(4, \mathbb{R})$. We explained in detail elsewhere
[1] that with the particular realisation of these operators considered here, this is simply the " $\frac{1}{4}, \frac{3}{4}$ representation of $S U(1,1)$." The operators $K_{+}^{(2)}, K_{-}^{(2)}$ and $K_{0}^{(2)}$ similarly close under commutation into the same subalgebra. The three operators $L_{+}, L_{-}$and $L_{0} \equiv K_{0}^{(1)}$ $+K_{0}^{(2)}$ also close into the same $S U(1,1)$ subalgebra, but we explained elsewhere [9] that in this case the particular realisation of (8) and (9) now leads to the "discrete series representation of $S U(1,1)$." Finally, the three operators $J_{+}, J_{-}$and $J_{0} \equiv K_{0}^{(1)}-K_{0}^{(2)}$ also close under commutation, in this case into (the Schwinger representation of) the angular momentum subalgebra of $S U(2)$.

We now consider a unitary representation of the group $S p(4, \mathbb{R})$ which is realised by the ten-parameter family of operators $\hat{\theta} \equiv \hat{\theta}\left(\omega \psi, r \phi, \rho_{1} \theta_{1} \lambda_{1}, \rho_{2} \theta_{2} \lambda_{2}\right)$, defined by,

$\hat{\theta} \equiv W_{2}(\omega, \psi) V_{2}(r, \phi) U_{2}^{(1)}\left(\rho_{1} \theta_{1} \lambda_{1}\right) U_{2}^{(2)}\left(\rho_{2} \theta_{2} \lambda_{2}\right)$

where,

$$
\begin{gathered}
W_{2}(\omega, \psi) \equiv \exp \left(-\frac{1}{2} \omega \mathrm{e}^{-\mathrm{i} \psi} a_{1}^{\dagger} a_{2}+\frac{1}{2} \omega \mathrm{e}^{\mathrm{i} \psi} a_{1} a_{2}^{\dagger}\right) ; \\
\omega, \psi \in \mathbb{R} \\
V_{2}(r, \phi) \equiv \exp \left(-\frac{1}{2} r \mathrm{e}^{-\mathrm{i} \phi} a_{1}^{\dagger} a_{2}^{\dagger}+\frac{1}{2} r \mathrm{e}^{\mathrm{i} \phi} a_{1} a_{2}\right) ; r, \phi \in \mathbb{R}
\end{gathered}
$$

$$
\begin{aligned}
U_{2}^{(j)}\left(\rho_{j} \theta_{j} \lambda_{j}\right) & \equiv \exp \left(-\frac{1}{4} \rho_{j} \mathrm{e}^{-\mathrm{i} \theta_{j}} a_{j}^{\dagger 2}\right. \\
& \left.+\frac{1}{4} \rho_{j} \mathrm{e}^{\mathrm{i} \theta_{j}} a_{j}^{2}\right) \exp \left(\mathrm{i} \lambda_{j} a_{j}^{\dagger} a_{j}\right) ;
\end{aligned}
$$

$\rho_{j}, \theta_{j}, \lambda_{j} \in \mathbb{R}, \quad j=1,2$.

As we have already explained, the following special cases of (11) and (12) apply: (i) for either $\rho_{2}=\lambda_{2}=r$ $=\omega=0$ or $\rho_{1}=\lambda_{1}=r=\omega=0$, we have the $\frac{1}{4}, \frac{3}{4}$ representation of $S U(1,1)$; (ii) for $\rho_{1}=\rho_{2}=\lambda_{1}-\lambda_{2}$ $=\omega=0$, we have the discrete series representations of $S U(1,1)$; and (iii) for $\rho_{1}=\rho_{2}=\lambda_{1}+\lambda_{2}=r=0$, we have the Schwinger representation of $S U(2)$.

We note next that it is not difficult to show that the mode of action of the unitary operators $\hat{\theta}$ on the basic operators $a_{i}$ and $a_{1}^{\dagger}(i=1,2)$ is as follows,

$\hat{\theta} a_{1} \hat{\theta}^{\dagger} \equiv b_{1}=\sigma_{1} a_{1}+\tau_{1} a_{1}^{\dagger}+\mu_{1} a_{2}+v_{1} a_{2}^{\dagger}$

$\hat{\theta} a_{1}^{\dagger} \hat{\theta}^{\dagger} \equiv b_{1}^{\dagger}=\tau_{1}^{*} a_{1}+\sigma_{1}^{*} a_{1}^{\dagger}+v_{1}^{*} a_{2}+\mu_{1}^{*} a_{2}^{\dagger}$

$\hat{\theta} a_{2} \hat{\theta}^{\dagger} \equiv b_{2}=\sigma_{2} a_{1}+\tau_{2} a_{1}^{\dagger}+\mu_{2} a_{2}+v_{2} a_{2}^{\dagger}$

$\hat{\theta} a_{2}^{\dagger} \hat{\theta}^{\dagger} \equiv b_{2}^{\dagger}=\tau_{2}^{*} a_{1}+\sigma_{2}^{*} a_{1}^{\dagger}+v_{2}^{*} a_{2}+\mu_{2}^{*} a_{2}^{\dagger}$

where the constants $\sigma_{i}, \tau_{i}, \mu_{i}$ and $v_{i}(i=1,2)$ are defined by,

$$
\begin{aligned}
\sigma_{1} \equiv & \mathrm{e}^{-\mathrm{i} \lambda_{1}} \cosh \left(\frac{1}{2} \rho_{1}\right) \cosh \left(\frac{1}{2} r\right) \cos \left(\frac{1}{2} \omega\right) \\
& -\mathrm{e}^{\mathrm{i}\left(\phi-\lambda_{1}-\theta_{1}+\psi\right)} \sinh \left(\frac{1}{2} \rho_{1}\right) \sinh \left(\frac{1}{2} r\right) \sin \left(\frac{1}{2} \omega\right) \\
\tau_{1} \equiv & \mathrm{e}^{-\mathrm{i}\left(\lambda_{1}+\theta_{1}\right)} \sinh \left(\frac{1}{2} \rho_{1}\right) \cosh \left(\frac{1}{2} r\right) \cos \left(\frac{1}{2} \omega\right) \\
& -\mathrm{e}^{-\mathrm{i}\left(\lambda_{1}+\phi+\psi\right)} \cosh \left(\frac{1}{2} \rho_{1}\right) \sinh \left(\frac{1}{2} r\right) \sin \left(\frac{1}{2} \omega\right)
\end{aligned}
$$




$$
\begin{aligned}
& \mu_{1} \equiv \mathrm{e}^{\mathrm{i}\left(\phi-\lambda_{1}-\theta_{1}\right)} \sinh \left(\frac{1}{2} \rho_{1}\right) \sinh \left(\frac{1}{2} r\right) \cos \left(\frac{1}{2} \omega\right) \\
& +\mathrm{e}^{-\mathrm{i}\left(\lambda_{1}+\psi\right)} \cosh \left(\frac{1}{2} \rho_{1}\right) \cosh \left(\frac{1}{2} r\right) \sin \left(\frac{1}{2} \omega\right) \\
& v_{1} \equiv \mathrm{e}^{-\mathrm{i}\left(\lambda_{1}+\phi\right)} \cosh \left(\frac{1}{2} \rho_{1}\right) \sinh \left(\frac{1}{2} r\right) \cos \left(\frac{1}{2} \omega\right) \\
& +\mathrm{e}^{\mathrm{i}\left(\psi-\lambda_{1}-\theta_{1}\right)} \sinh \left(\frac{1}{2} \rho_{1}\right) \cosh \left(\frac{1}{2} r\right) \sin \left(\frac{1}{2} \omega\right) \\
& \sigma_{2} \equiv \mathrm{e}^{\mathrm{i}\left(\phi-\lambda_{2}-\theta_{2}\right)} \sinh \left(\frac{1}{2} \rho_{2}\right) \sinh \left(\frac{1}{2} r\right) \cos \left(\frac{1}{2} \omega\right) \\
& -\mathrm{e}^{\mathrm{i}\left(\psi-\lambda_{2}\right)} \cosh \left(\frac{1}{2} \rho_{2}\right) \cosh \left(\frac{1}{2} r\right) \sin \left(\frac{1}{2} \omega\right) \\
& \tau_{2} \equiv \mathrm{e}^{-\mathrm{i}\left(\lambda_{2}+\phi\right)} \cosh \left(\frac{1}{2} \rho_{2}\right) \sinh \left(\frac{1}{2} r\right) \cos \left(\frac{1}{2} \omega\right) \\
& -\mathrm{e}^{-\mathrm{i}\left(\psi+\lambda_{2}+\theta_{2}\right)} \sinh \left(\frac{1}{2} \rho_{2}\right) \cosh \left(\frac{1}{2} r\right) \sin \left(\frac{1}{2} \omega\right) \\
& \mu_{2} \equiv \mathrm{e}^{-\mathrm{i} \lambda_{2}} \cosh \left(\frac{1}{2} \rho_{2}\right) \cosh \left(\frac{1}{2} r\right) \cos \left(\frac{1}{2} \omega\right) \\
& +\mathrm{e}^{\mathrm{i}\left(\phi-\lambda_{2}-\theta_{2}-\psi\right)} \sinh \left(\frac{1}{2} \rho_{2}\right) \sinh \left(\frac{1}{2} r\right) \sin \left(\frac{1}{2} \omega\right) \\
& v_{2} \equiv \mathrm{e}^{-\mathrm{i}\left(\lambda_{2}+\theta_{2}\right)} \sinh \left(\frac{1}{2} \rho_{2}\right) \cosh \left(\frac{1}{2} r\right) \cos \left(\frac{1}{2} \omega\right) \\
& +\mathrm{e}^{\mathrm{i}\left(\psi-\lambda_{2}-\phi\right)} \cosh \left(\frac{1}{2} \rho_{2}\right) \sinh \left(\frac{1}{2} r\right) \sin \left(\frac{1}{2} \omega\right) .
\end{aligned}
$$

Equations (13) and (14) provide us with the most general homogeneous linear transformations among the four operators $a_{1}, a_{1}^{\dagger}, a_{2}, a_{2}^{\dagger}$ that preserve the commutation relations (8),

$\left[b_{1}, b_{1}^{\dagger}\right]=1=\left[b_{2}, b_{2}^{\dagger}\right]$

$\left[b_{1}, b_{2}\right]=\left[b_{1}, b_{2}^{\dagger}\right]=\left[b_{1}^{\dagger}, b_{2}\right]=\left[b_{1}^{\dagger}, b_{2}^{\dagger}\right]=0$.

The operators $\hat{\theta}$ thus most completely generalise to two modes, the Bogoliubov transformation (5) for a single mode, which is in turn generated by the operator $U_{2}$.

From (13) and the fact that $\hat{\theta}$ is unitary, we may trivially prove that for an arbitrary function $f\left(a_{1}, a_{1}^{\dagger}, a_{2}, a_{2}^{\dagger}\right)$ we have,

$\hat{\theta} f\left(a_{1}, a_{1}^{\dagger}, a_{2}, a_{2}^{\dagger}\right) \hat{\theta}^{\dagger}=f\left(b_{1}, b_{1}^{\dagger}, b_{2}, b_{2}^{\dagger}\right) \Leftrightarrow$

$\hat{\theta} f\left(a_{1}, a_{1}^{\dagger}, a_{2}, a_{2}^{\dagger}\right)=f\left(b_{1}, b_{1}^{\dagger}, b_{2}, b_{2}^{\dagger}\right) \hat{\theta}$.

If we now define the general two-mode squeezed states as

$$
\left|A_{1} A_{2}\right\rangle_{\text {sq }} \equiv \hat{\theta}\left|A_{1} A_{2}\right\rangle,
$$

then (16) implies that $\hat{\theta} a_{1}=b_{1} \hat{\theta}$ and $\hat{\theta} a_{2}=b_{2} \hat{\theta}$, and hence that the states $\left|A_{1} A_{2}\right\rangle_{\text {sq }}$ are simultaneous eigenstates of the transformed operators $b_{1}$ and $b_{2}$,

$$
\begin{aligned}
& b_{1}\left|A_{1} A_{2}\right\rangle_{\mathrm{sq}}=A_{1}\left|A_{1} A_{2}\right\rangle_{\mathrm{sq}} \\
& b_{2}\left|A_{1} A_{2}\right\rangle_{\mathrm{sq}}=A_{2}\left|A_{1} A_{2}\right\rangle_{\mathrm{sq}} .
\end{aligned}
$$

We note also the relation,

$$
\begin{aligned}
\left|A_{1} A_{2}\right\rangle_{\mathrm{sq}}= & \hat{\theta} \exp \left(A_{1} a_{1}^{\dagger}-A_{1}^{*} a_{1}\right) \exp \left(A_{2} a_{2}^{\dagger}\right. \\
& \left.-A_{2}^{*} a_{2}\right)|0,0\rangle \\
= & \exp \left(A_{1} b_{1}^{\dagger}-A_{1}^{*} b_{1}\right) \exp \left(A_{2} b_{2}^{\dagger}-\right. \\
& \left.A_{2}^{*} b_{2}\right)|0,0\rangle_{\mathrm{sq}} .
\end{aligned}
$$

It is clear from (18) and (19) that the states $\left|A_{1} A_{2}\right\rangle_{\mathrm{sq}}$ are ordinary (Glauber) coherent states with respect to the transformed operators $b_{1}$ and $b_{2}$. As special cases of our general two-mode squeezed states, we mention the following:

(i) $r=\omega=0$, which corresponds to squeezing of the two modes independently;

(ii) $\rho_{1}=\rho_{2}=\lambda_{1}-\lambda_{2}=\omega=0$, which corresponds precisely to the squeezing considered by Caves and Schumaker [8]; and

(iii) $\rho_{1}=\rho_{2}=\lambda_{1}+\lambda_{2}=r=0$, which corresponds to $S U(2)$ squeezing.

In conclusion, it is clear that we have completely generalised and unified the various different types of two-mode squeezing that have been studied in the literature.

RFB gratefully acknowledges support for this work in the form of a research grant from the Science and Engineering Research Council of Great Britain. AV gratefully acknowledges support from GSI (Darmstadt).

\section{References}

1. Stoler, D.: Phys. Rev. Lett. 33, 1397 (1974)

2. Yuen, H.P.: Phys. Rev. A 13, 2226 (1976)

3. Caves, C.M.: Phys. Rev. D 26, 1817 (1982)

4. Walls, D.F.: Nature 306, 141 (1983)

5. Bishop, R.F., Vourdas, A.: J. Phys. A 19, 2525 (1986)

6. Schumaker, B.L.: Phys. Rep. 135, 317 (1986)

7. Milburn, G.J.: J. Phys. A 17, 737 (1984)

8. Caves, C.M., Schumaker, B.L.: Phys. Rev. A 31, 3068, 3093 (1985)

9. Bishop, R.F., Vourdas, A.: J. Phys. A 20, 3727 (1987)

10. Yurke, B., McCall, S.L., Klauder, J.R.: Phys. Rev. A 33, 4033 (1986)

11. Wodkiewicz, K., Eberly, J.H.: J. Opt. Soc. Am. B2, 458 (1985)

R.F. Bishop

Department of Mathematics

University of Manchester

Institute of Science and Technology

P.O. Box 88

Manchester M60 1QD

United Kingdom

A. Vourdas

Department of Electrical

Engineering and Electronics

University of Liverpool

Brownlow Hill

P.O. Box 147

Liverpool L69 3BX

United Kingdom 